\title{
A contribution to the study of the Lower Volga center of scarab beetle diversity in Russia: checklist of the tribe Aphodiini (Coleoptera, Scarabaeidae) of Dosang environs
}

\author{
Andrey Frolov ${ }^{\dagger}$, Lilia Akhmetova ${ }^{\dagger}$ \\ † Zoological Institute of Russian Academy of Sciences, Sankt-Petersburg, Russia
}

\section{Corresponding author: Andrey Frolov (aphodius@rambler.ru)}

Academic editor: Lyubomir Penev

Received: 02 Aug 2013 | Accepted: 29 Aug 2013 | Published: 16 Sep 2013

Citation: Frolov A, Akhmetova L (2013) A contribution to the study of the Lower Volga center of scarab beetle diversity in Russia: checklist of the tribe Aphodiini (Coleoptera, Scarabaeidae) of Dosang environs. Biodiversity Data Journal 1: e979. doi: 10.3897/BDJ.1.e979

\section{Abstract}

The field sampling of the Aphodiini scarab beetles in Dosang environs (Astrakhan Province, European Russia) in 2006-2012 resulted in the collection of 44 species. All but one of them belong to Aphodius Hellwig (sensu lato). This is apparently the richest recorded local Aphodiini fauna in Russia. The high Aphodiini diversity in the area can be explained by the long vegetative season with high effective heat sum, large livestock providing abundant food resources throughout the year, and location in the transition belt between Volga-Akhtuba Floodplain and Desert floristic districts. The core fauna consists of mesophilous species widely distributed in the Palearctic region and confined to the intrazonal habitats. Other species have ranges mostly limited to the steppe, semidesert, and desert zones.

\section{Keywords}

Scarab beetles, dung-beetles, aphodiines, Caspian lowland desert, faunistic composition 


\section{Introduction}

The tribe Aphodiini is the most diverse and widespread among the scarab beetles of the Northern Palearctic, including Russia. The members of this group dominate dung beetle communities and a few species can be found as far north as 65 degree of the northern latitude. However, like the majority of other scarabs, Aphodiini are the most diverse in the southern regions of the country. In Russia, major centers of aphodiine diversity are chiefly found in mountainous regions - Caucasus, Altai Mountains, Southern Primorye (Akhmetova 2011). The exception to this is the Caspian lowland desert with rich scarab beetle fauna (Nikolajev 1987, Shokhin 2007). Lower Volga, and especially Caspian lowland desert has long been recognized as a center of scarab beetle diversity in Russia. A number of first country records of aphodiines have originated from this area (Akhmetova and Frolov 2008b, Akhmetova and Frolov 2008a, Akhmetova and Frolov 2009) yet its fauna is still inadequately explored.

We have summarized the data of multi-year survey in Astrakhan Province and provided a check-list of the tribe Aphodiini of the Dosang environs. The list includes 44 species for which all but one of which belong to the mega-diverse genus Aphodius Hellwig (= Aphodius Illiger) (sensu lato). Notes on the distribution of the species and feeding preferences, if known, are given. The biogeographic composition of the fauna and possible reasons of high local species richness are discussed.

\section{Materials and methods}

\section{Study area}

The study area is situated on the Akhtuba River (fork of Volga River) about $90 \mathrm{~km}$ upstream of Volga estuary, Astrakhan Province, European Russia. We were based in Dosang Railway Station ( $46^{\circ} 54^{\prime} \mathrm{E} 47^{\circ} 55^{\prime}$ ). All species listed below and the majority of specimens were collected within $1 \mathrm{~km}$ from the station i.e. within an area of about $3 \mathrm{~km}^{2}$.

Four main biotopes were sampled: fixed sands (Fig. 1), barchan sands (Fig. 2), floodplain of Akhtuba River (Fig. 3), and riverside secondary forest (mainly of ash) (Fig. 4). 


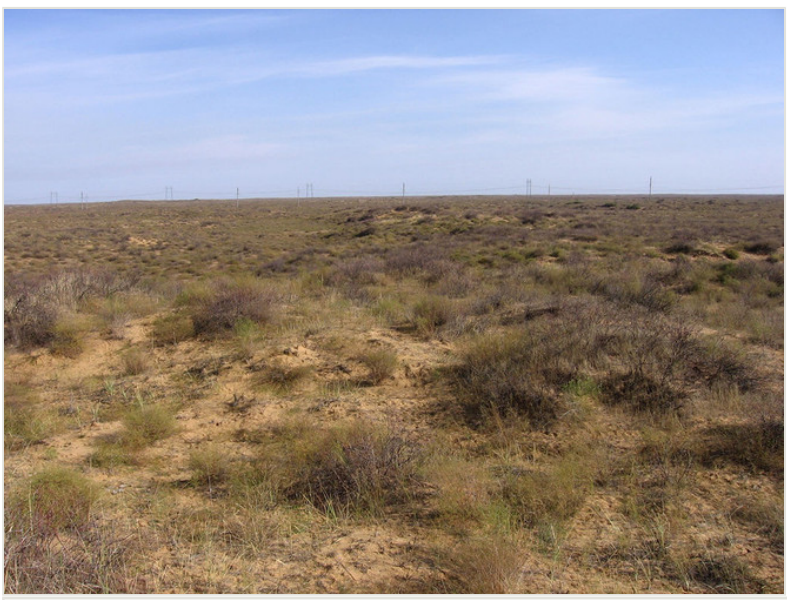

Figure 1.

Fixed sands near Dosang, Astrakhan Province, Russia.

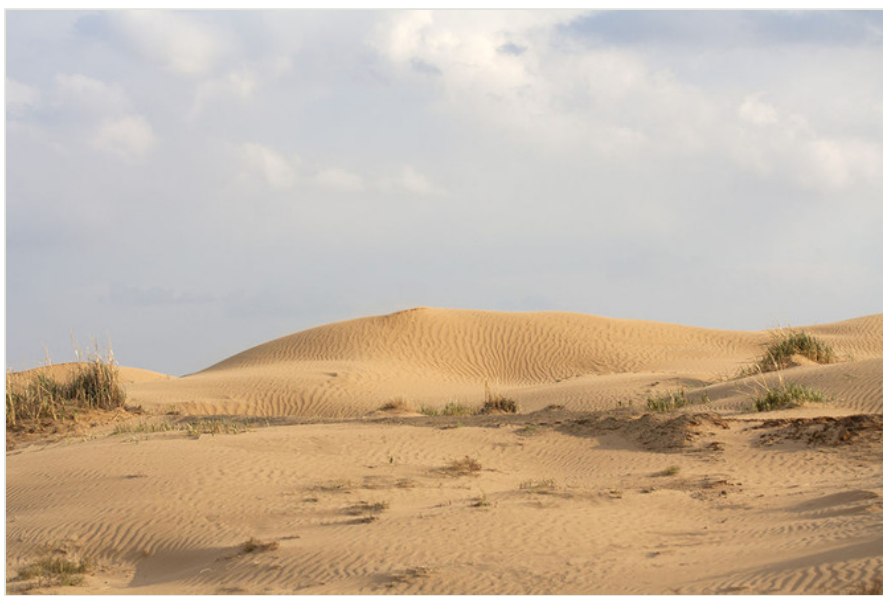

Figure 2.

Barchan sands North-East of Dosang, Astrakhan Province, Russia. 


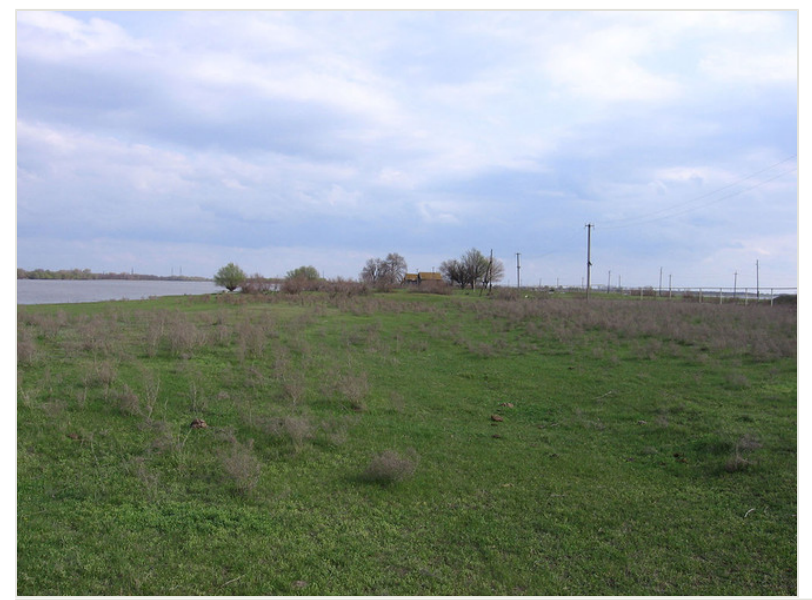

Figure 3.

Right side of Akhtuba River, floodplain, Dosang environs, Astrakhan Province, Russia.

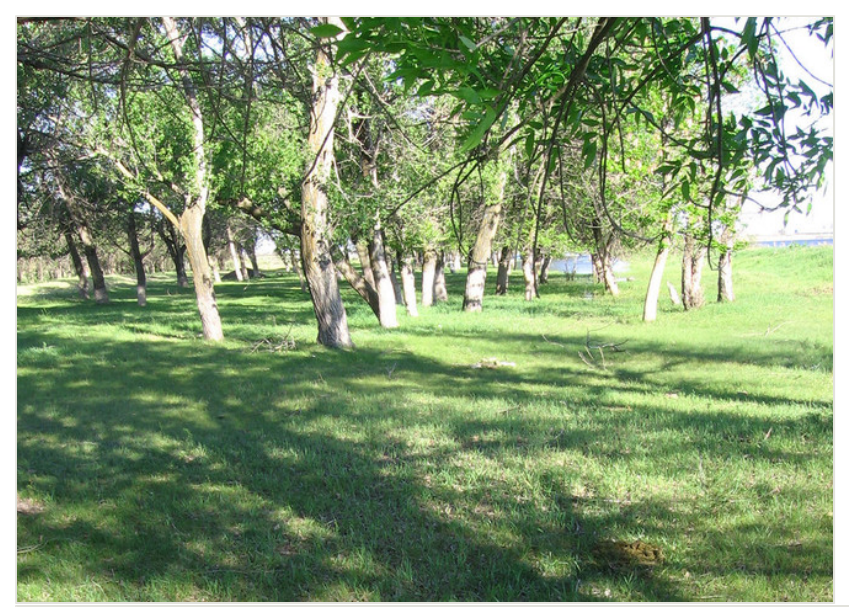

Figure 4.

Left side of Akhtuba River, riverine secondary forest near Dosang, Astrakhan Province, Russia.

\section{Material}

The material used in this work was collected by the authors from 2006 to 2012 in different seasons but primarily in spring and fall. Additional specimens were received from colleagues listed in the acknowledgement section. All the material mentioned is deposited in the collection of Zoological Institute of Russian Academy of Sciences, Sankt-Petersburg (ZIN). 


\section{Methods}

The beetles were collected by the following methods: washing of fresh horse and cow dung, hand collecting from fresh and old dung, mercury lamp traps, soil sifting with a sieve, hand collecting from marmot holes. The larvae were collected from old dung and soil and some specimens from each lot were reared to adults in the laboratory.

Classification and nomenclature of taxa follow Nikolajev (1987), Kabakov and Frolov (1996), Frolov (2002) and Akhmetova and Frolov (2008b). Classification of distribution ranges of Aphodiini species follows Akhmetova (2011) and is based on the zoogeographical divisions of the Palearctic Region (Emeljanov 1974). Aphodius species are arranged alphabetically by epithet.

\section{Checklist of the tribe Aphodiini of Dosang environs}

\section{Aphodius (Erytus) aequalis A. Schmidt, 1907}

Materials

a. country: Russia; stateProvince: Astrakhan'; locality: Dosang Railway Station; decimalLatitude: 46.90; decimalLongitude: 47.92; samplingProtocol: light trap; eventDate: 2007-05-23; individualCount: 10; recordedBy: A. V. Frolov, L. A. Akhmetova; collectionID: urn:Isid:biocol.org:col:34969; institutionCode: ZIN; collectionCode: Coleoptera

b. country: Russia; stateProvince: Astrakhan'; locality: Dosang environs, fixed sands; decimalLatitude: 46.92; decimalLongitude: 47.92; samplingProtocol: cow dung washing; eventDate: 2012-05-15; individualCount: 14; recordedBy: A. V. Frolov, L. A. Akhmetova; collectionID: urn:Isid:biocol.org:col:34969; institutionCode: ZIN; collectionCode: Coleoptera

Feeds on: Cattle dung, mostly cow dung.

Distribution: Southern Palearctic region up to Saur-Tarbagataj in the north-east.

\section{Aphodius (Chilothorax) badenkoi Nikolajev, 1987}

\section{Material}

a. country: Russia; stateProvince: Astrakhan'; locality: Dosang environs, fixed sands; decimalLatitude: 46.92; decimalLongitude: 47.92; samplingProtocol: horse dung washing; eventDate: 2007-04-17; individualCount: 1; recordedBy: A. V. Frolov, L. A. Akhmetova; collectionID: urn:Isid:biocol.org:col:34969; institutionCode: ZIN; collectionCode: Coleoptera

Feeds on: Cattle dung.

Distribution: Middle Asian deserts, Caspian lowland desert. 


\section{Aphodius (Acrossus) bimaculatus (Laxmann, 1770)}

\section{Materials}

a. country: Russia; stateProvince: Astrakhan'; locality: Dosang environs, fixed sands; decimalLatitude: 46.92; decimalLongitude: 47.92; samplingProtocol: horse dung washing; eventDate: 2007-04-17; individualCount: 2; recordedBy: A. V. Frolov, L. A. Akhmetova; collectionID: urn:Isid:biocol.org:col:34969; institutionCode: ZIN; collectionCode: Coleoptera

b. country: Russia; stateProvince: Astrakhan'; locality: Dosang environs, fixed sands; samplingProtocol: horse dung washing; eventDate: 2007-04-17; individualCount: 6; recordedBy: A. V. Frolov, L. A. Akhmetova; collectionID: urn:Isid:biocol.org:col:34969; institutionCode: ZIN; collectionCode: Coleoptera

c. country: Russia; stateProvince: Astrakhan'; locality: Dosang environs, fixed sands; decimalLatitude: 0.00; samplingProtocol: horse dung washing; eventDate: 2008-04-04; individualCount: 1; recordedBy: A. V. Frolov, L. A. Akhmetova; collectionID: urn:Isid:biocol.org:col:34969; institutionCode: ZIN; collectionCode: Coleoptera

d. country: Russia; stateProvince: Astrakhan'; locality: Dosang environs, left bank of Akhtuba River, floodplain; decimalLatitude: 46.91; decimalLongitude: 47.91; samplingProtocol: horse dung washing; eventDate: 2008-04-06; individualCount: 2; recordedBy: A. V. Frolov, L. A. Akhmetova; collectionID: urn:Isid:biocol.org:col:34969; institutionCode: ZIN; collectionCode: Coleoptera

Feeds on: Adults and larvae feed on horse dung (Fig. 5).

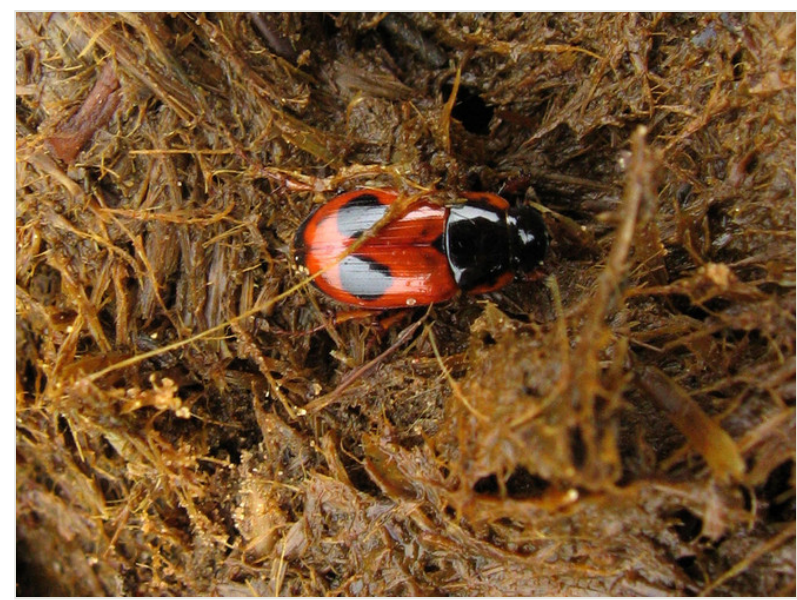

Figure 5.

Aphodius bimaculatus. Dosang environs, Astrakhan Province, Russia.

Distribution: Central and Eastern Europe, Western Asia up to East Kazakhstan in the east. The species is mostly occur in forest-steppe and steppe zones. 


\section{Aphodius (Melaphodius) caspius Ménétriés, 1832}

\section{Material}

a. country: Russia; stateProvince: Astrakhan'; locality: Dosang environs, left bank of Akhtuba River, floodplain; decimalLatitude: 46.91; decimalLongitude: 47.91; samplingProtocol: horse dung washing; eventDate: 2006-10-06; individualCount: 1; recordedBy: A. V. Frolov, L. A. Akhmetova; collectionID: urn:Isid:biocol.org:col:34969; institutionCode: ZIN; collectionCode: Coleoptera

Feeds on: Cattle dung.

Distribution: Steppe zone from Caucasus to West Siberia.

\section{Aphodius (Chilothorax) clathratus Reitter, 1892}

\section{Materials}

a. country: Russia; stateProvince: Astrakhan'; locality: Dosang environs, fixed sands; decimalLatitude: 46.92; decimalLongitude: 47.92; samplingProtocol: horse dung washing; eventDate: 2005-10-29; individualCount: 3; recordedBy: V. Kozlov; collectionID: urn:Isid:biocol.org:col:34969; institutionCode: ZIN; collectionCode: Coleoptera

b. country: Russia; stateProvince: Astrakhan'; locality: 7 km NNE of Dosang; decimalLatitude: 46.93; decimalLongitude: 48.00; samplingProtocol: horse dung washing; eventDate: 2005-10-31; individualCount: 6; recordedBy: V. Kozlov; collectionID: urn:Isid:biocol.org:col:34969; institutionCode: ZIN; collectionCode: Coleoptera

c. country: Russia; stateProvince: Astrakhan'; locality: 7 km NNE of Dosang; decimalLatitude: 46.93; decimalLongitude: 48.00; samplingProtocol: horse dung washing; eventDate: 2005-11-01; individualCount: 6; recordedBy: V. Kozlov; collectionID: urn:Isid:biocol.org:col:34969; institutionCode: ZIN; collectionCode: Coleoptera

d. country: Russia; stateProvince: Astrakhan'; locality: Dosang environs, fixed sands; decimalLatitude: 46.92; decimalLongitude: 47.92; samplingProtocol: old horse dung, larvae hand collecting; eventDate: 2008-04-09; individualCount: 3; recordedBy: A. V. Frolov, L. A. Akhmetova; collectionID: urn:Isid:biocol.org:col:34969; institutionCode: ZIN; collectionCode: Coleoptera

Feeds on: Adults and larvae feed on horse dung.

Distribution: The species was recorded from Caucasus, Turkey, Iran, and Middle Asia (Nikolajev 1987). The range of it is unclear since it is difficult to separate from the closely-related and very similar $A$. melanostictus. Some published records of $A$. clathratus apparently belong to $A$. melanostictus.

\section{Aphodius (Euorodalus) coenosus (Panzer, 1798)}

\section{Materials}

a. country: Russia; stateProvince: Astrakhan'; locality: Dosang environs, fixed sands; decimalLatitude: 46.92; decimalLongitude: 47.92; samplingProtocol: horse dung washing; eventDate: 2007-04-17; individualCount: 1; recordedBy: A. V. Frolov, L. A. Akhmetova; 
collectionID: urn:Isid:biocol.org:col:34969; institutionCode: ZIN; collectionCode:

Coleoptera

b. country: Russia; stateProvince: Astrakhan'; locality: 15 km NE of Dosang; decimalLatitude: 47.00; decimalLongitude: 47.98; samplingProtocol: horse dung washing; eventDate: 2008-05-07; individualCount: 1; recordedBy: A. V. Frolov, L. A. Akhmetova; collectionID: urn:Isid:biocol.org:col:34969; institutionCode: ZIN; collectionCode:

Coleoptera

c. country: Russia; stateProvince: Astrakhan'; locality: Dosang environs, left bank of Akhtuba River, floodplain; decimalLatitude: 46.91; decimalLongitude: 47.91; samplingProtocol: horse dung washing; eventDate: 2008-05-07; individualCount: 1; recordedBy: A. V. Frolov, L. A. Akhmetova; collectionID: urn:Isid:biocol.org:col:34969; institutionCode: ZIN; collectionCode: Coleoptera

Feeds on: Cattle and wild herbivore dung.

Distribution: Europe (except for northernmost part), Asia Minor.

\section{Aphodius (Mendidius) curtulus (Harold, 1866)}

\section{Materials}

a. country: Russia; stateProvince: Astrakhan'; locality: 15 km NE of Dosang; decimalLatitude: 47.00; decimalLongitude: 47.98; samplingProtocol: sand sifting; eventDate: 2008-04-07; individualCount: 2; recordedBy: A. V. Frolov, L. A. Akhmetova; collectionID: urn:Isid:biocol.org:col:34969; institutionCode: ZIN; collectionCode: Coleoptera

b. country: Russia; stateProvince: Astrakhan'; locality: Dosang environs, fixed sands; decimalLatitude: 46.92; decimalLongitude: 47.92; samplingProtocol: sand sifting; eventDate: 2006-10-7/11; individualCount: 3; recordedBy: A. V. Frolov, L. A. Akhmetova; collectionID: urn:Isid:biocol.org:col:34969; institutionCode: ZIN; collectionCode: Coleoptera

c. country: Russia; stateProvince: Astrakhan'; locality: Dosang environs, fixed sands; decimalLatitude: 46.92; decimalLongitude: 47.92; samplingProtocol: sand sifting; eventDate: 2007-04-13/15; individualCount: 1; recordedBy: A. V. Frolov, L. A. Akhmetova; collectionID: urn:Isid:biocol.org:col:34969; institutionCode: ZIN; collectionCode: Coleoptera

d. country: Russia; stateProvince: Astrakhan'; locality: 8 km NE of Dosang; decimalLatitude: 46.94; decimalLongitude: 48.02; samplingProtocol: sand sifting; eventDate: 2007-04-17/21; individualCount: 5; recordedBy: A. V. Frolov, L. A. Akhmetova; collectionID: urn:Isid:biocol.org:col:34969; institutionCode: ZIN; collectionCode: Coleoptera

Feeds on: Adults were found in sand near plant roots.

Distribution: Caspian lowland desert.

\section{Aphodius (Alocoderus) digitalis D. Koshantschikov, 1894}

\section{Material}

a. country: Russia; stateProvince: Astrakhan'; locality: Dosang Railway Station; decimalLatitude: 46.90; decimalLongitude: 47.92; samplingProtocol: light trap; eventDate: 
2007-05-23; individualCount: 1; recordedBy: A. V. Frolov, L. A. Akhmetova; collectionID: urn:Isid:biocol.org:col:34969; institutionCode: ZIN; collectionCode: Coleoptera

Distribution: Middle Asian deserts, Caspian lowland desert.

\section{Aphodius (Chilothorax) distinctus (Müller, 1776)}

\section{Materials}

a. country: Russia; stateProvince: Astrakhan'; locality: Dosang environs, left bank of Akhtuba River, floodplain; decimalLatitude: 46.91; decimalLongitude: 47.91; sampling Protocol: horse dung washing; eventDate: 2006-10-06; individualCount: 1; recordedBy: A. V. Frolov, L. A. Akhmetova; collectionID: urn:Isid:biocol.org:col:34969; institutionCode: ZIN; collectionCode: Coleoptera

b. country: Russia; stateProvince: Astrakhan'; locality: Dosang environs, left bank of Akhtuba River, floodplain; decimalLatitude: 46.91; decimalLongitude: 47.91; samplingProtocol: horse dung washing; eventDate: 2006-10-07; individualCount: 15; recordedBy: A. V. Frolov, L. A. Akhmetova; collectionID: urn:Isid:biocol.org:col:34969; institutionCode: ZIN; collectionCode: Coleoptera

c. country: Russia; stateProvince: Astrakhan'; locality: Dosang environs, fixed sands; decimalLatitude: 46.92; decimalLongitude: 47.92; samplingProtocol: horse dung washing; eventDate: 2006-10-09; individualCount: 9; recordedBy: A. V. Frolov, L. A. Akhmetova; collectionID: urn:Isid:biocol.org:col:34969; institutionCode: ZIN; collectionCode: Coleoptera

d. country: Russia; stateProvince: Astrakhan'; locality: Dosang environs, fixed sands; decimalLatitude: 46.92; decimalLongitude: 47.92; samplingProtocol: horse dung washing; eventDate: 2007-04-17; individualCount: 10; recordedBy: A. V. Frolov, L. A. Akhmetova; collectionID: urn:Isid:biocol.org:col:34969; institutionCode: ZIN; collectionCode:

Coleoptera

e. country: Russia; stateProvince: Astrakhan'; locality: 15 km NE of Dosang; decimalLatitude: 47.00; decimalLongitude: 47.98; samplingProtocol: cow dung washing; eventDate: 2010-10-12; individualCount: 1; recordedBy: A. V. Frolov; collectionID: urn:Isid:biocol.org:col:34969; institutionCode: ZIN; collectionCode: Coleoptera

Feeds on: Adults and larvae feed on cattle dung.

Distribution: The species is distributed throughout Europe, Transcaucasia, Central Asia, Northern Africa; it was introduced to North America.

\section{Aphodius (Nobius) dosangi Frolov et Akhmetova, 2008}

\section{Materials}

Holotype:

a. country: Russia; stateProvince: Astrakhan'; locality: Dosang environs, fixed sands; decimalLatitude: 46.90; decimalLongitude: 47.92; samplingProtocol: light trap; eventDate: 2007-05-24; individualCount: 1; sex: male; recordedBy: A. V. Frolov, L. A. Akhmetova; collectionID: urn:Isid:biocol.org:col:34969; institutionCode: ZIN; collectionCode: Coleoptera

\section{Paratypes:}

a. country: Russia; stateProvince: Astrakhan'; locality: Dosang environs, fixed sands; decimalLatitude: 46.90; decimalLongitude: 47.92; samplingProtocol: light trap; eventDate: 
2007-05-24; individualCount: 5; sex: females; recordedBy: A. V. Frolov, L. A. Akhmetova; collectionID: urn:Isid:biocol.org:col:34969; institutionCode: ZIN; collectionCode: Coleoptera

b. country: Russia; stateProvince: Astrakhan'; locality: Dosang environs, left bank of Akhtuba River, floodplain; decimalLatitude: 46.91; decimalLongitude: 47.91; samplingProtocol: cow dung washing; eventDate: 2006-10-06; individualCount: 2; sex: females; recordedBy: A. V. Frolov, L. A. Akhmetova; collectionID: urn:Isid:biocol.org:col:34969; institutionCode: ZIN; collectionCode: Coleoptera

c. country: Russia; stateProvince: Astrakhan'; locality: Dosang environs, fixed sands; decimalLatitude: 46.90; decimalLongitude: 47.92; samplingProtocol: horse dung washing; eventDate: 2006-10-11; individualCount: 2; sex: 1 male and 1 female; recordedBy: A. V. Frolov, L. A. Akhmetova; collectionID: urn:Isid:biocol.org:col:34969; institutionCode: ZIN; collectionCode: Coleoptera

d. country: Russia; stateProvince: Astrakhan'; locality: Dosang environs, fixed sands; decimalLatitude: 46.92; decimalLongitude: 47.92; samplingProtocol: hand collecting from old horse dung; eventDate: 2007-04-13/15; individualCount: 1; sex: female; recordedBy: A. V. Frolov, L. A. Akhmetova; collectionID: urn:Isid:biocol.org:col:34969; institutionCode: ZIN; collectionCode: Coleoptera

e. country: Russia; stateProvince: Astrakhan'; locality: Dosang environs, fixed sands; decimalLatitude: 46.92; decimalLongitude: 47.92; samplingProtocol: larvae hand collecting old horse dung; eventDate: 2007-04-17; individualCount: 21; sex: 12 males and 9 females; recordedBy: A. V. Frolov, L. A. Akhmetova; collectionID: urn:Isid:biocol.org:col:34969; institutionCode: ZIN; collectionCode: Coleoptera

\section{Other materials:}

a. country: Russia; stateProvince: Astrakhan'; locality: Dosang environs, left bank of Akhtuba River, floodplain; decimalLatitude: 46.91; decimalLongitude: 47.91; samplingProtocol: horse dung washing; eventDate: 2007-04-10; individualCount: 2; recordedBy: A. V. Frolov, L. A. Akhmetova; collectionID: urn:Isid:biocol.org:col:34969; institutionCode: ZIN; collectionCode: Coleoptera

b. country: Russia; stateProvince: Astrakhan'; locality: Dosang environs, fixed sands; decimalLatitude: 46.92; decimalLongitude: 47.92; samplingProtocol: horse dung washing; eventDate: 2007-04-17; individualCount: 3; recordedBy: A. V. Frolov, L. A. Akhmetova; collectionID: urn:Isid:biocol.org:col:34969; institutionCode: ZIN; collectionCode: Coleoptera

c. country: Russia; stateProvince: Astrakhan'; locality: 8 km NE of Dosang; decimalLatitude: 46.94; decimalLongitude: 48.02; samplingProtocol: larvae hand collecting old horse dung; eventDate: 2008-04-09; individualCount: 12; recordedBy: A. V. Frolov, L. A. Akhmetova; collectionID: urn:Isid:biocol.org:col:34969; institutionCode: ZIN; collectionCode: Coleoptera

d. country: Russia; stateProvince: Astrakhan'; locality: Dosang environs, fixed sands; decimalLatitude: 46.92; decimalLongitude: 47.92; samplingProtocol: cow dung washing; eventDate: 2012-05-15; individualCount: 3; recordedBy: A. V. Frolov, L. A. Akhmetova; collectionID: urn:Isid:biocol.org:col:34969; institutionCode: ZIN; collectionCode: Coleoptera

Feeds on: Adults were found in horse and cow dung.

Distribution: Caspian lowland desert. 


\section{Aphodius (Colobopterus) erraticus (Linnaeus, 1758)}

\section{Material}

a. country: Russia; stateProvince: Astrakhan'; locality: Dosang environs, left bank of Akhtuba River, floodplain; decimalLatitude: 46.91; decimalLongitude: 47.91; samplingProtocol: horse dung washing; eventDate: 2012-05-13; individualCount: 1; recordedBy: A. V. Frolov, L. A. Akhmetova; collectionID: urn:Isid:biocol.org:col:34969; institutionCode: ZIN; collectionCode: Coleoptera

Feeds on: Cattle and wild herbivore dung.

Distribution: Transpalearctic species, introduced to North America.

\section{Aphodius (Aphodius) fimetarius (Linnaeus, 1758)}

\section{Material}

a. country: Russia; stateProvince: Astrakhan'; locality: Dosang environs, fixed sands; decimalLatitude: 46.92; decimalLongitude: 47.92; samplingProtocol: horse dung washing; eventDate: 2006-10-09; individualCount: 2; recordedBy: A. V. Frolov, L. A. Akhmetova; collectionID: urn:Isid:biocol.org:col:34969; institutionCode: ZIN; collectionCode: Coleoptera

Feeds on: Cattle and wild herbivore dung.

Distribution: Transpalearctic species, introduced in North America and Australia.

\section{Aphodius (Loraspis) frater Mulsant et Rey, 1870}

\section{Materials}

a. country: Russia; stateProvince: Astrakhan'; locality: Dosang environs, fixed sands; decimalLatitude: 46.92; decimalLongitude: 47.92; samplingProtocol: hand collecting from old horse dung; eventDate: 2007-04-16; individualCount: 3; recordedBy: A. V. Frolov, L. A. Akhmetova; collectionID: urn:Isid:biocol.org:col:34969; institutionCode: ZIN; collectionCode: Coleoptera

b. country: Russia; stateProvince: Astrakhan'; locality: Dosang environs, left bank of Akhtuba River, floodplain; decimalLatitude: 46.91; decimalLongitude: 47.91; samplingProtocol: hand collecting from old horse dung; eventDate: 2008-04-03; individualCount: 15; recordedBy: A. V. Frolov, L. A. Akhmetova; collectionID: urn:Isid:biocol.org:col:34969; institutionCode: ZIN; collectionCode: Coleoptera

c. country: Russia; stateProvince: Astrakhan'; locality: Dosang environs, fixed sands; decimalLatitude: 46.92; decimalLongitude: 47.92; samplingProtocol: hand collecting from old horse dung; eventDate: 2007-04-13/15; individualCount: 2; recordedBy: A. V. Frolov, L. A. Akhmetova; collectionID: urn:Isid:biocol.org:col:34969; institutionCode: ZIN; collectionCode: Coleoptera

Feeds on: Adults feed on dry cattle dung.

Distribution: Western Palearctic region South Europe the south-west to West Siberia in the north-east. 


\section{Aphodius (Calamosternus) granarius (Linnaeus, 1767)}

\section{Materials}

a. country: Russia; stateProvince: Astrakhan'; locality: Dosang environs, left bank of Akhtuba River, floodplain; decimalLatitude: 46.91; decimalLongitude: 47.91; samplingProtocol: horse dung washing; eventDate: 2007-04-10; individualCount: 6; recordedBy: A. V. Frolov, L. A. Akhmetova; collectionID: urn:Isid:biocol.org:col:34969; institutionCode: ZIN; collectionCode: Coleoptera

b. country: Russia; stateProvince: Astrakhan'; locality: Dosang environs, right bank of Akhtuba River, floodplain; decimalLatitude: 46.90; decimalLongitude: 47.90; samplingProtocol: horse dung washing; eventDate: 2007-05-12; individualCount: 6; recordedBy: A. V. Frolov, L. A. Akhmetova; collectionID: urn:Isid:biocol.org:col:34969; institutionCode: ZIN; collectionCode: Coleoptera

c. country: Russia; stateProvince: Astrakhan'; locality: Dosang environs, left bank of Akhtuba River, floodplain; decimalLatitude: 46.91; decimalLongitude: 47.91; samplingProtocol: horse dung washing; eventDate: 2008-05-05; individualCount: 1; recordedBy: A. V. Frolov, L. A. Akhmetova; collectionID: urn:Isid:biocol.org:col:34969; institutionCode: ZIN; collectionCode: Coleoptera

d. country: Russia; stateProvince: Astrakhan'; locality: 15 km NE of Dosang; decimalLatitude: 47.00; decimalLongitude: 47.98; samplingProtocol: horse dung washing; eventDate: 2008-05-07; individualCount: 2; recordedBy: A. V. Frolov, L. A. Akhmetova; collectionID: urn:Isid:biocol.org:col:34969; institutionCode: ZIN; collectionCode: Coleoptera

Feeds on: Coprophagous generalist.

Distribution: West Palearctic region (except for northernmost part), introduced in North America.

\section{Aphodius (Bodilus) gregarius Harold, 1871}

\section{Material}

a. scientificName: gregarius; country: Russia; stateProvince: Astrakhan'; locality: Dosang Railway Station; decimalLatitude: 46.90; decimalLongitude: 47.92; samplingProtocol: light trap; eventDate: 2007-05-23; individualCount: 1; recordedBy: A. V. Frolov, L. A. Akhmetova; collectionID: urn:Isid:biocol.org:col:34969; institutionCode: ZIN; collectionCode: Coleoptera

Feeds on: Cattle dung.

Distribution: Steppe and semidesert zones from Lower Volga in the west to Mongolia in the east.

\section{Aphodius (Nobius) gresseri Semenov, 1898}

\section{Materials}

a. country: Russia; stateProvince: Astrakhan'; locality: Dosang environs, fixed sands; decimalLatitude: 46.91; decimalLongitude: 47.91; samplingProtocol: horse dung washing; eventDate: 2007-04-10; individualCount: 2; recordedBy: A. V. Frolov, L. A. Akhmetova; 
collectionID: urn:Isid:biocol.org:col:34969; institutionCode: ZIN; collectionCode: Coleoptera

b. country: Russia; stateProvince: Astrakhan'; locality: 1.5-5 km NE of Dosang; samplingProtocol: horse dung washing; eventDate: 2007-04-14; individualCount: 1; recordedBy: K. V. Makarov; collectionID: urn:Isid:biocol.org:col:34969; institutionCode: ZIN; collectionCode: Coleoptera

C. country: Russia; stateProvince: Astrakhan'; locality: Dosang environs, fixed sands; decimalLatitude: 46.92; decimalLongitude: 47.92; samplingProtocol: larvae hand collecting old horse dung; eventDate: 2007-04-16; individualCount: 2; recordedBy: A. V. Frolov, L. A. Akhmetova; collectionID: urn:Isid:biocol.org:col:34969; institutionCode: ZIN; collectionCode: Coleoptera

d. country: Russia; stateProvince: Astrakhan'; locality: Dosang environs, fixed sands; decimalLatitude: 46.92; decimalLongitude: 47.92; samplingProtocol: horse dung washing; eventDate: 2007-04-17; individualCount: 2; recordedBy: A. V. Frolov, L. A. Akhmetova; collectionID: urn:Isid:biocol.org:col:34969; institutionCode: ZIN; collectionCode: Coleoptera

e. country: Russia; stateProvince: Astrakhan'; locality: Dosang environs, fixed sands; decimalLatitude: 46.92; decimalLongitude: 47.92; samplingProtocol: larvae hand collecting old horse dung; eventDate: 2007-04-17; individualCount: 10; recordedBy: A. V. Frolov, L. A. Akhmetova; collectionID: urn:Isid:biocol.org:col:34969; institutionCode: ZIN; collectionCode: Coleoptera

f. country: Russia; stateProvince: Astrakhan'; locality: Dosang environs, fixed sands; decimalLatitude: 46.92; decimalLongitude: 47.92; samplingProtocol: larvae hand collecting old horse dung; eventDate: 2007-04-21; individualCount: 2; recordedBy: A. V. Frolov, L. A. Akhmetova; collectionID: urn:Isid:biocol.org:col:34969; institutionCode: ZIN; collectionCode: Coleoptera

Feeds on: Adults and larvae feed on cattle dung.

Distribution: Volga Region up to Vladimir Province in the north. Kazakh steppe up to Sultankeldy Lake in the East.

\section{Aphodius (Sugrames) hauseri (Reitter, 1894)}

\section{Materials}

a. country: Russia; stateProvince: Astrakhan'; locality: 2 km NE of Dosang; decimalLatitude: 46.92; decimalLongitude: 47.93; samplingProtocol: larvae hand collecting from sand; eventDate: 2007-05-01; individualCount: 5; recordedBy: A. V. Frolov, L. A. Akhmetova; collectionID: urn:Isid:biocol.org:col:34969; institutionCode: ZIN; collectionCode: Coleoptera

b. country: Russia; stateProvince: Astrakhan'; locality: 15 km NE of Dosang; decimalLatitude: 47.00; decimalLongitude: 47.98; samplingProtocol: sand sifting; eventDate: 2008-05-07; individualCount: 2; recordedBy: A. V. Frolov, L. A. Akhmetova; collectionID: urn:Isid:biocol.org:col:34969; institutionCode: ZIN; collectionCode: Coleoptera

Distribution: Middle Asian deserts. 


\section{Aphodius (Alocoderus) hydrochaeris (Fabricius, 1798)}

\section{Materials}

a. country: Russia; stateProvince: Astrakhan'; locality: Dosang environs, left bank of Akhtuba River, floodplain; decimalLatitude: 46.91; decimalLongitude: 47.91; samplingProtocol: cow dung washing; eventDate: 2006-10-06; individualCount: 3; recordedBy: A. V. Frolov, L. A. Akhmetova; collectionID: urn:Isid:biocol.org:col:34969; institutionCode: ZIN; collectionCode: Coleoptera

b. country: Russia; stateProvince: Astrakhan'; locality: Dosang Railway Station; decimalLatitude: 46.90; decimalLongitude: 47.92; samplingProtocol: light trap; eventDate: 2007-05-23; individualCount: 1; recordedBy: A. V. Frolov, L. A. Akhmetova; collectionID: urn:Isid:biocol.org:col:34969; institutionCode: ZIN; collectionCode: Coleoptera

c. country: Russia; stateProvince: Astrakhan'; locality: Dosang environs, left bank of Akhtuba River, floodplain; decimalLatitude: 46.91; decimalLongitude: 47.91; samplingProtocol: horse dung washing; eventDate: 2012-05-13; individualCount: 1; recordedBy: A. V. Frolov, L. A. Akhmetova; collectionID: urn:Isid:biocol.org:col:34969; institutionCode: ZIN; collectionCode: Coleoptera

Feeds on: Horse dung.

Distribution: West Palearctic region (except for northernmost part).

\section{Aphodius (Bodilus) ictericus (Laicharting, 1781)}

\section{Materials}

a. country: Russia; stateProvince: Astrakhan'; locality: Dosang environs, left bank of Akhtuba River, floodplain; decimalLatitude: 46.91; decimalLongitude: 47.91; samplingProtocol: cow dung washing; eventDate: 2006-10-06; individualCount: 4; recordedBy: A. V. Frolov, L. A. Akhmetova; collectionID: urn:Isid:biocol.org:col:34969; institutionCode: ZIN; collectionCode: Coleoptera

b. country: Russia; stateProvince: Astrakhan'; locality: Dosang environs, left bank of Akhtuba River, floodplain; decimalLatitude: 46.91; decimalLongitude: 47.91; samplingProtocol: cow dung washing; eventDate: 2006-10-07; individualCount: 10; recordedBy: A. V. Frolov, L. A. Akhmetova; collectionID: urn:Isid:biocol.org:col:34969; institutionCode: ZIN; collectionCode: Coleoptera

c. country: Russia; stateProvince: Astrakhan'; locality: Dosang environs, fixed sands; decimalLatitude: 46.92; decimalLongitude: 47.92; samplingProtocol: cow dung washing; eventDate: 2012-05-15; individualCount: 34; recordedBy: A. V. Frolov, L. A. Akhmetova; collectionID: urn:Isid:biocol.org:col:34969; institutionCode: ZIN; collectionCode: Coleoptera

Feeds on: Cattle dung.

Distribution: Distributed throughout Europe (except for northernmost part), in North Africa, Asia Minor, Iran, up to Irtysh River in the east. 


\section{Aphodius (Acanthobodilus) immundus Creutzer, 1799}

\section{Materials}

a. country: Russia; stateProvince: Astrakhan'; locality: Dosang environs, left bank of Akhtuba River, floodplain; decimalLatitude: 46.91; decimalLongitude: 47.91; samplingProtocol: cow dung washing; eventDate: 2006-10-06; individualCount: 10; recordedBy: A. V. Frolov, L. A. Akhmetova; collectionID: urn:Isid:biocol.org:col:34969; institutionCode: ZIN; collectionCode: Coleoptera

b. country: Russia; stateProvince: Astrakhan'; locality: Dosang environs, fixed sands; decimalLatitude: 46.92; decimalLongitude: 47.92; samplingProtocol: cow dung washing; eventDate: 2007-04-16; individualCount: 10; recordedBy: A. V. Frolov, L. A. Akhmetova; collectionID: urn:Isid:biocol.org:col:34969; institutionCode: ZIN; collectionCode: Coleoptera

c. country: Russia; stateProvince: Astrakhan'; locality: Dosang Railway Station; decimalLatitude: 46.90; decimalLongitude: 47.92; samplingProtocol: light trap; eventDate: 2007-05-23; individualCount: 4; recordedBy: A. V. Frolov, L. A. Akhmetova; collectionID: urn:Isid:biocol.org:col:34969; institutionCode: ZIN; collectionCode: Coleoptera

d. country: Russia; stateProvince: Astrakhan'; locality: Dosang environs, left bank of Akhtuba River, floodplain; decimalLatitude: 46.91; decimalLongitude: 47.91; samplingProtocol: horse dung washing; eventDate: 2012-05-13; individualCount: 1; recordedBy: A. V. Frolov, L. A. Akhmetova; collectionID: urn:Isid:biocol.org:col:34969; institutionCode: ZIN; collectionCode: Coleoptera

e. country: Russia; stateProvince: Astrakhan'; locality: Dosang environs, fixed sands; decimalLatitude: 46.92; decimalLongitude: 47.92; samplingProtocol: cow dung washing; eventDate: 2012-05-15; individualCount: 2; recordedBy: A. V. Frolov, L. A. Akhmetova; collectionID: urn:Isid:biocol.org:col:34969; institutionCode: ZIN; collectionCode:

Coleoptera

Feeds on: Cattle dung

Distribution: Western Palearctic region from North Africa in the south-west to Baikal Lake in the north-east.

\section{Aphodius (Aphodaulacus) kizeritskyi Frolov, 2002}

\section{Materials}

a. country: Russia; stateProvince: Astrakhan'; locality: 7 km NNE of Dosang; samplingProtocol: horse dung washing; eventDate: 2005-10-31; individualCount: 22; recordedBy: V. Kozlov; collectionID: urn:Isid:biocol.org:col:34969; institutionCode: ZIN; collectionCode: Coleoptera

b. country: Russia; stateProvince: Astrakhan'; locality: 7 km NNE of Dosang; decimalLatitude: 46.93; decimalLongitude: 48.00; samplingProtocol: horse dung washing; eventDate: 2005-11-01; individualCount: 35; recordedBy: V. Kozlov; collectionID: urn:Isid:biocol.org:col:34969; institutionCode: ZIN; collectionCode: Coleoptera

c. country: Russia; stateProvince: Astrakhan'; locality: Dosang environs, fixed sands; decimalLatitude: 46.92; decimalLongitude: 47.92; samplingProtocol: horse dung washing; eventDate: 2005-11-01; individualCount: 36; recordedBy: A. V. Frolov; collectionID: urn:Isid:biocol.org:col:34969; institutionCode: ZIN; collectionCode: Coleoptera 
d. country: Russia; stateProvince: Astrakhan'; locality: 15 km NE of Dosang; decimalLatitude: 47.00; decimalLongitude: 47.98; samplingProtocol: horse dung washing; eventDate: 2009-10-31; individualCount: 48; recordedBy: A. V. Frolov; collectionID: urn:Isid:biocol.org:col:34969; institutionCode: ZIN; collectionCode: Coleoptera

e. country: Russia; stateProvince: Astrakhan'; locality: 8 km NE of Dosang; decimalLatitude: 46.94; decimalLongitude: 48.02; samplingProtocol: horse dung washing; eventDate: 2009-11-04; individualCount: 9; recordedBy: A. V. Frolov; collectionID: urn:Isid:biocol.org:col:34969; institutionCode: ZIN; collectionCode: Coleoptera

Feeds on: Horse dung.

Distribution: Caspian lowland desert, Middle Asian deserts.

\section{Aphodius (Mecynodes) kisilkumi Solsky, 1876}

\section{Material}

a. country: Russia; stateProvince: Astrakhan'; locality: Dosang environs, fixed sands; decimalLatitude: 46.92; decimalLongitude: 47.92; samplingProtocol: cow dung washing; eventDate: 2012-05-15; individualCount: 16; recordedBy: A. V. Frolov, L. A. Akhmetova; collectionID: urn:Isid:biocol.org:col:34969; institutionCode: ZIN; collectionCode: Coleoptera

Feeds on: Cattle dung.

Distribution: Caspian lowland desert, Middle Asian deserts.

\section{Aphodius (Liothorax) kraatzi Harold, 1868}

\section{Materials}

a. country: Russia; stateProvince: Astrakhan'; locality: Dosang Railway Station; decimalLatitude: 46.90; decimalLongitude: 47.92; samplingProtocol: light trap; eventDate: 2007-05-23; individualCount: 1; recordedBy: A. V. Frolov, L. A. Akhmetova; collectionID: urn:Isid:biocol.org:col:34969; institutionCode: ZIN; collectionCode: Coleoptera

b. country: Russia; stateProvince: Astrakhan'; locality: Dosang Railway Station; decimalLatitude: 46.90; decimalLongitude: 47.92; samplingProtocol: light trap; eventDate: 2007-05-23; individualCount: 16; recordedBy: A. V. Frolov, L. A. Akhmetova; collectionID: urn:Isid:biocol.org:col:34969; institutionCode: ZIN; collectionCode: Coleoptera

Feeds on: Apparently saprophagous species.

Distribution: Distributed mostly in steppe and desert zones in South Europe, Caucasus, Asia Minor, Middle Asia.

\section{Aphodius (Labarrus) lividus (Olivier, 1789)}

\section{Materials}

a. country: Russia; stateProvince: Astrakhan'; locality: Dosang Railway Station; decimalLatitude: 46.90; decimalLongitude: 47.92; samplingProtocol: light trap; eventDate: 2007-05-23; individualCount: 12; recordedBy: A. V. Frolov, L. A. Akhmetova; collectionID: urn:Isid:biocol.org:col:34969; institutionCode: ZIN; collectionCode: Coleoptera 
b. country: Russia; stateProvince: Astrakhan'; locality: 15 km NE of Dosang; decimalLatitude: 47.00; decimalLongitude: 47.98; samplingProtocol: horse dung washing; eventDate: 2008-05-07; individualCount: 1; recordedBy: A. V. Frolov, L. A. Akhmetova; collectionID: urn:Isid:biocol.org:col:34969; institutionCode: ZIN; collectionCode: Coleoptera

c. country: Russia; stateProvince: Astrakhan'; locality: Dosang environs, fixed sands; decimalLatitude: 46.92; decimalLongitude: 47.92; samplingProtocol: cow dung washing; eventDate: 2012-05-15; individualCount: 15; recordedBy: A. V. Frolov, L. A. Akhmetova; collectionID: urn:Isid:biocol.org:col:34969; institutionCode: ZIN; collectionCode: Coleoptera

Feeds on: Cattle dung.

Distribution: West Palearctic region (except for northernmost part).

\section{Aphodius (Bodilus) lugens Creutzer, 1799}

\section{Materials}

a. country: Russia; stateProvince: Astrakhan'; locality: Dosang environs, left bank of Akhtuba River, floodplain; decimalLatitude: 46.91; decimalLongitude: 47.91; samplingProtocol: cow dung washing; eventDate: 2006-10-06; individualCount: 3; recordedBy: A. V. Frolov, L. A. Akhmetova; collectionID: urn:Isid:biocol.org:col:34969; institutionCode: ZIN; collectionCode: Coleoptera

b. country: Russia; stateProvince: Astrakhan'; locality: Dosang environs, fixed sands; decimalLatitude: 46.92; decimalLongitude: 47.92; samplingProtocol: cow dung washing; eventDate: 2012-05-15; individualCount: 1; recordedBy: A. V. Frolov, L. A. Akhmetova; collectionID: urn:Isid:biocol.org:col:34969; institutionCode: ZIN; collectionCode: Coleoptera

Feeds on: Cattle dung.

Distribution: Western Palearctic region from North Africa in the south-west to SaurTarbagataj in the north-east.

\section{Aphodius (Chilothorax) melanostictus W. Schmidt, 1840}

\section{Materials}

a. country: Russia; stateProvince: Astrakhan'; locality: 7 km NNE of Dosang; decimalLatitude: 46.93; decimalLongitude: 48.00; samplingProtocol: horse dung washing; eventDate: 2005-11-01; individualCount: 25; recordedBy: V. Kozlov; collectionID: urn:Isid:biocol.org:col:34969; institutionCode: ZIN; collectionCode: Coleoptera

b. country: Russia; stateProvince: Astrakhan'; locality: Dosang environs, fixed sands; decimalLatitude: 46.92; decimalLongitude: 47.92; samplingProtocol: horse dung washing; eventDate: 2006-10-09; individualCount: 4; recordedBy: A. V. Frolov, L. A. Akhmetova; collectionID: urn:Isid:biocol.org:col:34969; institutionCode: ZIN; collectionCode: Coleoptera

c. country: Russia; stateProvince: Astrakhan'; locality: Dosang environs, fixed sands; decimalLatitude: 46.92; decimalLongitude: 47.92; samplingProtocol: horse dung washing; eventDate: 2007-04-17; individualCount: 15; recordedBy: A. V. Frolov, L. A. Akhmetova; collectionID: urn:Isid:biocol.org:col:34969; institutionCode: ZIN; collectionCode: Coleoptera 
Feeds on: Cattle dung.

Distribution: Western Palearctic region up to South Siberia in the north-east.

\section{Aphodius (Mendidius) multiplex Reitter, 1897}

\section{Materials}

a. country: Russia; stateProvince: Astrakhan'; locality: Dosang environs, fixed sands; decimalLatitude: 46.92; decimalLongitude: 47.92; samplingProtocol: horse dung washing; eventDate: 2007-04-17; individualCount: 9; recordedBy: A. V. Frolov, L. A. Akhmetova; collectionID: urn:Isid:biocol.org:col:34969; institutionCode: ZIN; collectionCode:

Coleoptera

b. country: Russia; stateProvince: Astrakhan'; locality: Dosang environs, fixed sands; decimalLatitude: 46.92; decimalLongitude: 47.92; samplingProtocol: horse dung washing; eventDate: 2012-05-15; individualCount: 11; recordedBy: A. V. Frolov, L. A. Akhmetova; collectionID: urn:Isid:biocol.org:col:34969; institutionCode: ZIN; collectionCode: Coleoptera

c. country: Russia; stateProvince: Astrakhan'; locality: Dosang environs, fixed sands; decimalLatitude: 46.92; decimalLongitude: 47.92; samplingProtocol: horse dung washing; eventDate: 2007-04-13/15; individualCount: 6; recordedBy: A. V. Frolov, L. A. Akhmetova; collectionID: urn:Isid:biocol.org:col:34969; institutionCode: ZIN; collectionCode:

Coleoptera

Feeds on: Cattle dung.

Distribution: Steppe and semidesert zones from Ciscaucasia in the west to Mongolia in the east.

\section{Aphodius (Chilothorax) plustschewskii D. Koshantschikov, 1894}

\section{Materials}

a. country: Russia; stateProvince: Astrakhan'; locality: 7 km NNE of Dosang; samplingProtocol: manually collected from horse dung; eventDate: 2005-10-31; individualCount: 2; recordedBy: V. Kozlov; collectionID: urn:Isid:biocol.org:col:34969; institutionCode: ZIN; collectionCode: Coleoptera

b. country: Russia; stateProvince: Astrakhan'; locality: Dosang environs, fixed sands; decimalLatitude: 46.92; decimalLongitude: 47.92; samplingProtocol: manually collected from horse dung; eventDate: 2005-11-01; individualCount: 37; recordedBy: A. V. Frolov; collectionID: urn:Isid:biocol.org:col:34969; institutionCode: ZIN; collectionCode: Coleoptera

c. country: Russia; stateProvince: Astrakhan'; locality: 6 km NNE of Dosang; samplingProtocol: manually collected from horse dung; eventDate: 2005-11-02; individualCount: 2; recordedBy: V. Kozlov; collectionID: urn:Isid:biocol.org:col:34969; institutionCode: ZIN; collectionCode: Coleoptera

d. country: Russia; stateProvince: Astrakhan'; locality: 7 km NNE of Dosang; samplingProtocol: manually collected from horse dung; eventDate: 2005-11-03; individualCount: 2; recordedBy: V. Kozlov; collectionID: urn:Isid:biocol.org:col:34969; institutionCode: ZIN; collectionCode: Coleoptera

e. country: Russia; stateProvince: Astrakhan'; locality: 15 km NE of Dosang; decimalLatitude: 47.00; decimalLongitude: 47.98; samplingProtocol: manually collected 
from horse dung; eventDate: 2009-10-31; individualCount: 1; recordedBy: A. V. Frolov; collectionID: urn:Isid:biocol.org:col:34969; institutionCode: ZIN; collectionCode: Coleoptera

f. country: Russia; stateProvince: Astrakhan'; locality: Dosang environs, fixed sands; samplingProtocol: manually collected from horse dung; eventDate: 2009-11-02; individualCount: 88; recordedBy: A. V. Frolov; collectionID: urn:Isid:biocol.org:col:34969; institutionCode: ZIN; collectionCode: Coleoptera

Feeds on: Horse dung (Fig. 6).

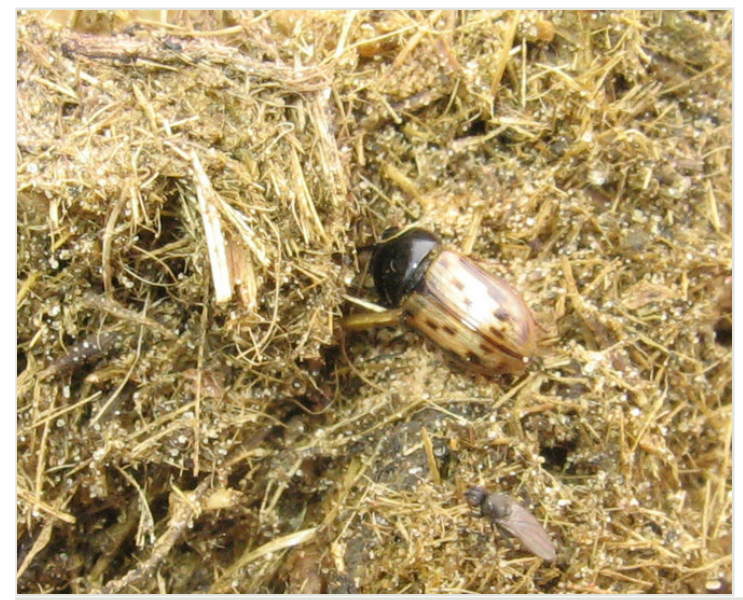

Figure 6.

Aphodius plustschewskii in horse dung. Dosang environs, Astrakhan Province, Russia.

Distribution: Caspian lowland desert, Middle Asian deserts

\section{Aphodius (Bodilus) punctipennis Erichson, 1848}

\section{Material}

a. country: Russia; stateProvince: Astrakhan'; locality: Dosang Railway Station; decimalLatitude: 46.90; decimalLongitude: 47.92; samplingProtocol: light trap; eventDate: 2007-05-23; individualCount: 1; recordedBy: A. V. Frolov, L. A. Akhmetova; collectionID: urn:Isid:biocol.org:col:34969; institutionCode: ZIN; collectionCode: Coleoptera

Feeds on: Cattle dung

Distribution: Southern Central Europe, Transcaucasus, Caspian lowland and Central Asian Deserts up to Dzhungar Alatau in the east

\section{Aphodius (Eudolus) quadriguttatus (Herbst, 1783)}

\section{Materials}

a. country: Russia; stateProvince: Astrakhan'; locality: Dosang environs, fixed sands; decimalLatitude: 46.92; decimalLongitude: 47.92; samplingProtocol: cow dung washing; eventDate: 2007-04-17; individualCount: 6; recordedBy: A. V. Frolov, L. A. Akhmetova; 
collectionID: urn:Isid:biocol.org:col:34969; institutionCode: ZIN; collectionCode: Coleoptera

b. country: Russia; stateProvince: Astrakhan'; locality: Dosang environs, fixed sands; decimalLatitude: 46.92; decimalLongitude: 47.92; samplingProtocol: cow dung washing; eventDate: 2012-05-15; individualCount: 12; recordedBy: A. V. Frolov, L. A. Akhmetova; collectionID: urn:Isid:biocol.org:col:34969; institutionCode: ZIN; collectionCode:

Coleoptera

Feeds on: Cattle and wild herbivore dung.

Distribution: Western Palearctic region from North Africa in the south-west to Altai Mountains the north-east.

\section{Aphodius (Acrossus) rufipes (Linnaeus, 1758)}

\section{Material}

a. country: Russia; stateProvince: Astrakhan'; locality: Dosang environs, left bank of Akhtuba River, floodplain; decimalLatitude: 46.91; decimalLongitude: 47.91; samplingProtocol: cow dung washing; eventDate: 2006-10-06; individualCount: 1; recordedBy: A. V. Frolov, L. A. Akhmetova; collectionID: urn:Isid:biocol.org:col:34969; institutionCode: ZIN; collectionCode: Coleoptera

Feeds on: Cattle and wild herbivore dung.

Distribution: Transpalearctic species.

\section{Aphodius (Biralus) satellitius (Herbst, 1789)}

\section{Materials}

a. country: Russia; stateProvince: Astrakhan'; locality: Dosang environs, fixed sands; decimalLatitude: 46.92; decimalLongitude: 47.92; samplingProtocol: horse dung washing; eventDate: 2007-04-17; individualCount: 2; recordedBy: A. V. Frolov, L. A. Akhmetova; collectionID: urn:Isid:biocol.org:col:34969; institutionCode: ZIN; collectionCode: Coleoptera

b. country: Russia; stateProvince: Astrakhan'; locality: Dosang environs, right bank of Akhtuba River, floodplain; decimalLatitude: 46.90; decimalLongitude: 47.90; samplingProtocol: horse dung washing; eventDate: 2007-05-23; individualCount: 14; recordedBy: A. V. Frolov, L. A. Akhmetova; collectionID: urn:Isid:biocol.org:col:34969; institutionCode: ZIN; collectionCode: Coleoptera

c. country: Russia; stateProvince: Astrakhan'; locality: Dosang environs, left bank of Akhtuba River, floodplain; decimalLatitude: 46.91; decimalLongitude: 47.91; samplingProtocol: horse dung washing; eventDate: 2012-05-13; individualCount: 1; recordedBy: A. V. Frolov, L. A. Akhmetova; collectionID: urn:Isid:biocol.org:col:34969; institutionCode: ZIN; collectionCode: Coleoptera

d. country: Russia; stateProvince: Astrakhan'; locality: Dosang environs, fixed sands; decimalLatitude: 46.92; decimalLongitude: 47.92; samplingProtocol: horse dung washing; eventDate: 2007-04-13/15; individualCount: 1; recordedBy: A. V. Frolov, L. A. Akhmetova; collectionID: urn:Isid:biocol.org:col:34969; institutionCode: ZIN; collectionCode:

Coleoptera

Feeds on: Cattle dung. 
Distribution: Western Palearctic region from North Africa in the south-west to southern Urals in the north-east. Mostly in steppe zones.

\section{Aphodius (Trichonotulus) scrofa (Fabricius, 1787)}

\section{Material}

a. country: Russia; stateProvince: Astrakhan'; locality: Dosang environs, left bank of Akhtuba River, floodplain; decimalLatitude: 46.91; decimalLongitude: 47.91; samplingProtocol: horse dung washing; eventDate: 2007-04-10; individualCount: 4; recordedBy: A. V. Frolov, L. A. Akhmetova; collectionID: urn:Isid:biocol.org:col:34969; institutionCode: ZIN; collectionCode: Coleoptera

Feeds on: Cattle and wild herbivore dung.

Distribution: Transpalearctic species occurring mostly in steppe zone.

\section{Aphodius (Nobius) serotinus (Panzer, 1799)}

\section{Materials}

a. country: Russia; stateProvince: Astrakhan'; locality: Dosang environs, left bank of Akhtuba River, floodplain; decimalLatitude: 46.91; decimalLongitude: 47.91; samplingProtocol: cow dung washing; eventDate: 2006-10-06; individualCount: 3; recordedBy: A. V. Frolov, L. A. Akhmetova; collectionID: urn:Isid:biocol.org:col:34969; institutionCode: ZIN; collectionCode: Coleoptera

b. country: Russia; stateProvince: Astrakhan'; locality: Dosang environs, left bank of Akhtuba River, floodplain; decimalLatitude: 46.91; decimalLongitude: 47.91; samplingProtocol: cow dung washing; eventDate: 2006-10-07; individualCount: 18; recordedBy: A. V. Frolov, L. A. Akhmetova; collectionID: urn:Isid:biocol.org:col:34969; institutionCode: ZIN; collectionCode: Coleoptera

c. country: Russia; stateProvince: Astrakhan'; locality: Dosang environs, fixed sands; decimalLatitude: 46.92; decimalLongitude: 47.92; samplingProtocol: larvae hand collecting old horse dung; eventDate: 2007-04-17; individualCount: 8; recordedBy: A. V. Frolov, L. A. Akhmetova; collectionID: urn:Isid:biocol.org:col:34969; institutionCode: ZIN; collectionCode: Coleoptera

d. country: Russia; stateProvince: Astrakhan'; locality: Dosang environs, left bank of Akhtuba River, floodplain; decimalLatitude: 46.91; decimalLongitude: 47.91; samplingProtocol: larvae hand collecting old horse dung; eventDate: 2007-05-17; individualCount: 9; recordedBy: A. V. Frolov, L. A. Akhmetova; collectionID: urn:Isid:biocol.org:col:34969; institutionCode: ZIN; collectionCode: Coleoptera

e. country: Russia; stateProvince: Astrakhan'; locality: Dosang environs, left bank of Akhtuba River, floodplain; decimalLatitude: 46.91; decimalLongitude: 47.91; samplingProtocol: larvae hand collecting old horse dung; eventDate: 2007-05-18; individualCount: 8; recordedBy: A. V. Frolov, L. A. Akhmetova; collectionID: urn:Isid:biocol.org:col:34969; institutionCode: ZIN; collectionCode: Coleoptera

Feeds on: Adults were found in horse and cow dung, larvae in horse dung.

Distribution: Widely distributed in Europe and Asia up to Baikal Lake in the east. 


\section{Aphodius (Subrinus) sturmi Harold, 1870}

\section{Materials}

a. country: Russia; stateProvince: Astrakhan'; locality: Dosang environs, fixed sands; decimalLatitude: 46.92; decimalLongitude: 47.92; samplingProtocol: horse dung washing; eventDate: 2006-10-09; individualCount: 1; recordedBy: A. V. Frolov, L. A. Akhmetova; collectionID: urn:Isid:biocol.org:col:34969; institutionCode: ZIN; collectionCode: Coleoptera

b. country: Russia; stateProvince: Astrakhan'; locality: Dosang environs, left bank of Akhtuba River, floodplain; decimalLatitude: 46.91; decimalLongitude: 47.91; samplingProtocol: larvae hand collecting old horse dung; eventDate: 2007-05-17; individualCount: 7; recordedBy: A. V. Frolov, L. A. Akhmetova; collectionID: urn:Isid:biocol.org:col:34969; institutionCode: ZIN; collectionCode: Coleoptera

c. country: Russia; stateProvince: Astrakhan'; locality: Dosang environs, fixed sands; decimalLatitude: 46.92; decimalLongitude: 47.92; samplingProtocol: cow dung washing; eventDate: 2012-05-15; individualCount: 8; recordedBy: A. V. Frolov, L. A. Akhmetova; collectionID: urn:Isid:biocol.org:col:34969; institutionCode: ZIN; collectionCode: Coleoptera

Feeds on: Cattle dung, mostly cow dung.

Distribution: Transpalearctic species, mostly in steppe and semidesert zone.

\section{Aphodius (Eupleurus) subterraneus (Linnaeus, 1758)}

\section{Materials}

a. country: Russia; stateProvince: Astrakhan'; locality: Dosang environs, fixed sands; decimalLatitude: 46.92; decimalLongitude: 47.92; samplingProtocol: cow dung washing; eventDate: 2007-04-17; individualCount: 4; recordedBy: A. V. Frolov, L. A. Akhmetova; collectionID: urn:Isid:biocol.org:col:34969; institutionCode: ZIN; collectionCode: Coleoptera

b. country: Russia; stateProvince: Astrakhan'; locality: Dosang environs, right bank of Akhtuba River, floodplain; decimalLatitude: 46.90; decimalLongitude: 47.90; samplingProtocol: larvae hand collecting old horse dung; eventDate: 2007-05-24; individualCount: 8; recordedBy: A. V. Frolov, L. A. Akhmetova; collectionID: urn:Isid:biocol.org:col:34969; institutionCode: ZIN; collectionCode: Coleoptera

c. country: Russia; stateProvince: Astrakhan'; locality: Dosang environs, left bank of Akhtuba River, floodplain; decimalLatitude: 46.91; decimalLongitude: 47.91; samplingProtocol: horse dung washing; eventDate: 2008-05-05; individualCount: 1; recordedBy: A. V. Frolov, L. A. Akhmetova; collectionID: urn:Isid:biocol.org:col:34969; institutionCode: ZIN; collectionCode: Coleoptera

Feeds on: Cattle dung.

Distribution: Distributed throughout Palaearctic region (except for northernmost part), introduced in North America. 


\section{Aphodius (Heptaulacus) sus (Herbst, 1783)}

\section{Materials}

a. country: Russia; stateProvince: Astrakhan'; locality: Dosang environs, left bank of Akhtuba River, floodplain; decimalLatitude: 46.91; decimalLongitude: 47.91; samplingProtocol: horse dung washing; eventDate: 2006-10-06; individualCount: 1; recordedBy: A. V. Frolov, L. A. Akhmetova; collectionID: urn:Isid:biocol.org:col:34969; institutionCode: ZIN; collectionCode: Coleoptera

b. country: Russia; stateProvince: Astrakhan'; locality: Dosang environs, left bank of Akhtuba River, floodplain; decimalLatitude: 46.91; decimalLongitude: 47.91; samplingProtocol: horse dung washing; eventDate: 2006-10-07; individualCount: 18; recordedBy: A. V. Frolov, L. A. Akhmetova; collectionID: urn:Isid:biocol.org:col:34969; institutionCode: ZIN; collectionCode: Coleoptera

c. country: Russia; stateProvince: Astrakhan'; locality: S of Dosang, near Akhtuba River floodplain; samplingProtocol: larvae hand collecting from soil; eventDate: 2007-05-01; individualCount: 6; recordedBy: A. V. Frolov, L. A. Akhmetova; collectionID: urn:Isid:biocol.org:col:34969; institutionCode: ZIN; collectionCode: Coleoptera

Feeds on: Adults occur in large numbers in horse dung in the fall. Larvae were collected among grass roots at the depth of $10-15 \mathrm{~cm}$ in riverine secondary forest (Frolov 2009).

Distribution: Middle and southern Europe, Minor Asia, North Africa, northern Iran, Central Asia up to South Siberia.

\section{Aphodius (Heptaulacus) testudinarius (Fabricius, 1775)}

\section{Materials}

a. country: Russia; stateProvince: Astrakhan'; locality: Dosang environs, Akhtuba River floodplain; decimalLatitude: 46.91; decimalLongitude: 47.91; samplingProtocol: hand collecting from old horse dung; eventDate: 2007-04-10; individualCount: 4; recordedBy: A. V. Frolov, L. A. Akhmetova; collectionID: urn:Isid:biocol.org:col:34969; institutionCode: ZIN; collectionCode: Coleoptera

b. country: Russia; stateProvince: Astrakhan'; locality: Dosang environs, fixed sands; decimalLatitude: 46.92; decimalLongitude: 47.92; samplingProtocol: hand collecting from old horse dung; eventDate: 2007-04-17; individualCount: 4; recordedBy: A. V. Frolov, L. A. Akhmetova; collectionID: urn:Isid:biocol.org:col:34969; institutionCode: ZIN; collectionCode: Coleoptera

c. country: Russia; stateProvince: Astrakhan'; locality: Dosang environs, fixed sands; decimalLatitude: 46.92; decimalLongitude: 47.92; samplingProtocol: hand collecting from old horse dung; eventDate: 2007-04-13/15; individualCount: 16; recordedBy: A. V. Frolov, L. A. Akhmetova; collectionID: urn:Isid:biocol.org:col:34969; institutionCode: ZIN; collectionCode: Coleoptera

Feeds on: Adults occur in dry cattle dung.

Distribution: Distributed mostly in steppe zone from South Europe to Central Kazakhstan. 


\section{Aphodius (Nialus) varians Duftschmidt, 1805}

\section{Materials}

a. country: Russia; stateProvince: Astrakhan'; locality: Dosang Railway Station; decimalLatitude: 46.90; decimalLongitude: 47.92; samplingProtocol: light trap; eventDate: 2007-05-23; individualCount: 7; recordedBy: A. V. Frolov, L. A. Akhmetova; collectionID: urn:Isid:biocol.org:col:34969; institutionCode: ZIN; collectionCode: Coleoptera

b. country: Russia; stateProvince: Astrakhan'; locality: Dosang environs, left bank of Akhtuba River, floodplain; decimalLatitude: 46.91; decimalLongitude: 47.91; samplingProtocol: horse dung washing; eventDate: 2008-05-05; individualCount: 5; recordedBy: A. V. Frolov, L. A. Akhmetova; collectionID: urn:Isid:biocol.org:col:34969; institutionCode: ZIN; collectionCode: Coleoptera

c. country: Russia; stateProvince: Astrakhan'; locality: Dosang environs, fixed sands; decimalLatitude: 46.92; decimalLongitude: 47.92; samplingProtocol: cow dung washing; eventDate: 2012-05-15; individualCount: 1; recordedBy: A. V. Frolov, L. A. Akhmetova; collectionID: urn:Isid:biocol.org:col:34969; institutionCode: ZIN; collectionCode:

Coleoptera

Feeds on: Adults feed on cattle dung.

Distribution: Widely distributed in western Palaearctic region up to Zaissan Lake in the east.

\section{Aphodius (Chilothorax) variicolor D. Koshantschikov, 1895}

\section{Materials}

a. country: Russia; stateProvince: Astrakhan'; locality: Dosang environs, fixed sands; eventDate: 1996-09-13; individualCount: 2; recordedBy: K. A. Grebennikov; collectionID: urn:Isid:biocol.org:col:34969; institutionCode: ZIN; collectionCode: Coleoptera

b. country: Russia; stateProvince: Astrakhan'; locality: 15 km NE of Dosang; decimalLatitude: 47.00; decimalLongitude: 47.98; samplingProtocol: larvae hand collecting from soil; eventDate: 2008-04-07; individualCount: 4; recordedBy: A. V. Frolov, L. A. Akhmetova; collectionID: urn:Isid:biocol.org:col:34969; institutionCode: ZIN; collectionCode: Coleoptera

c. country: Russia; stateProvince: Astrakhan'; locality: 15 km NE of Dosang; decimalLatitude: 47.00; decimalLongitude: 47.98; samplingProtocol: cow dung washing; eventDate: 2010-10-12; individualCount: 10; recordedBy: A. V. Frolov; collectionID: urn:Isid:biocol.org:col:34969; institutionCode: ZIN; collectionCode: Coleoptera

d. country: Russia; stateProvince: Astrakhan'; locality: Dosang environs, fixed sands; decimalLatitude: 46.92; decimalLongitude: 47.92; samplingProtocol: cow dung washing; eventDate: 2012-05-15; individualCount: 1; recordedBy: A. V. Frolov, L. A. Akhmetova; collectionID: urn:Isid:biocol.org:col:34969; institutionCode: ZIN; collectionCode: Coleoptera

e. country: Russia; stateProvince: Astrakhan'; locality: Dosang environs, fixed sands; decimalLatitude: 46.92; decimalLongitude: 47.92; samplingProtocol: cow dung washing; eventDate: 2006-10-7/11; individualCount: 3; recordedBy: A. V. Frolov, L. A. Akhmetova; collectionID: urn:Isid:biocol.org:col:34969; institutionCode: ZIN; collectionCode:

Coleoptera 
Feeds on: Adults feed on horse dung (Fig. 7), larvae were collected among the roots of cheat grass (Frolov 2009) (Fig. 8).

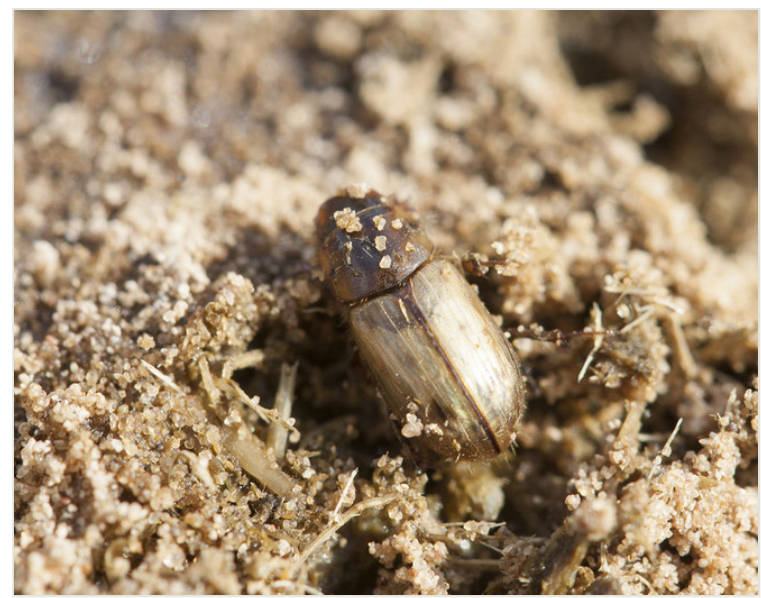

Figure 7.

Aphodius variicolor. Dosang environs, Astrakhan Province, Russia.

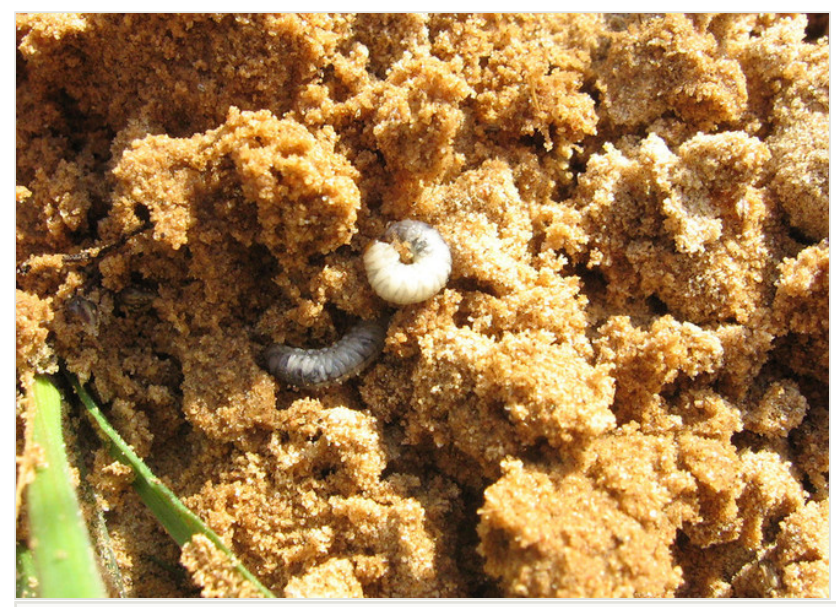

Figure 8.

Aphodius variicolor, 3-instar larvae. Dosang environs, Astrakhan Province, Russia.

Distribution: Caspian lowland desert.

\section{Aphodius (Orodaliscus) zangi A. Schmidt, 1906}

\section{Materials}

a. country: Russia; stateProvince: Astrakhan'; locality: Dosang environs, right bank of Akhtuba River, floodplain; decimalLatitude: 46.90; decimalLongitude: 47.90; 
samplingProtocol: hand collecting from marmot hole; eventDate: 2007-04-22; individualCount: 30; recordedBy: A. V. Frolov, L. A. Akhmetova; collectionID: urn:Isid:biocol.org:col:34969; institutionCode: ZIN; collectionCode: Coleoptera

b. country: Russia; stateProvince: Astrakhan'; locality: Dosang environs, right bank of Akhtuba River, floodplain; decimalLatitude: 46.90; decimalLongitude: 47.90; samplingProtocol: hand collecting from marmot hole; eventDate: 2008-04-3/9; individualCount: 47; recordedBy: A. V. Frolov, L. A. Akhmetova; collectionID: urn:Isid:biocol.org:col:34969; institutionCode: ZIN; collectionCode: Coleoptera

Feeds on: Rodent nest dweller, adults and apparently larvae feed on rodent excrement (Fig. 9).

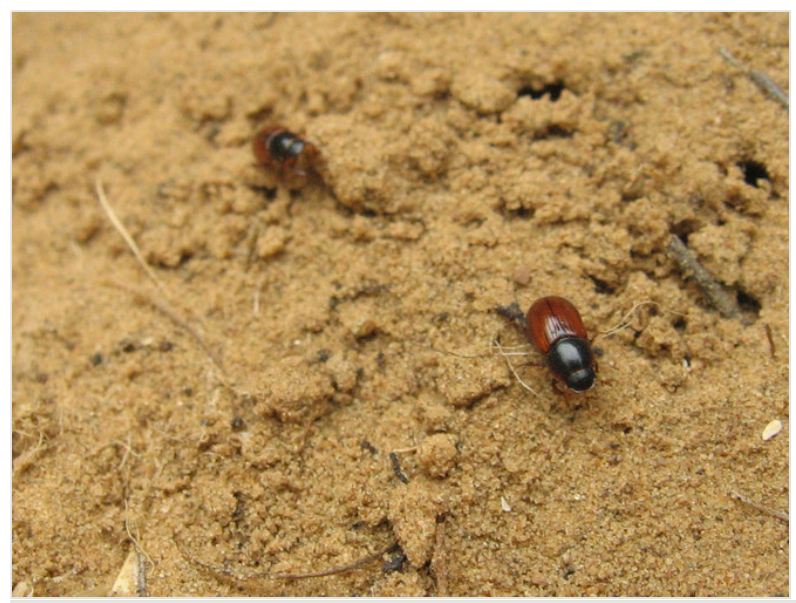

Figure 9.

Aphodius zangi clambering out of a marmot hole. Dosang environs, Astrakhan Province, Russia.

Distribution: Distributed mostly in the steppe and semidesert zones from Lower Volga in the west to West Siberia in the east.

\section{Aphodius (Planolinus) vittatus Say, 1825}

\section{Materials}

a. country: Russia; stateProvince: Astrakhan'; locality: Dosang environs, left bank of Akhtuba River, floodplain; decimalLatitude: 46.91; decimalLongitude: 47.91; samplingProtocol: cow dung washing; eventDate: 2007-05-21; individualCount: 15; recordedBy: A. V. Frolov, L. A. Akhmetova; collectionID: urn:Isid:biocol.org:col:34969; institutionCode: ZIN; collectionCode: Coleoptera

b. country: Russia; stateProvince: Astrakhan'; locality: Dosang environs, fixed sands; decimalLatitude: 46.92; decimalLongitude: 47.92; samplingProtocol: cow dung washing; eventDate: 2012-05-15; individualCount: 5; recordedBy: A. V. Frolov, L. A. Akhmetova; collectionID: urn:Isid:biocol.org:col:34969; institutionCode: ZIN; collectionCode: Coleoptera

c. country: Russia; stateProvince: Astrakhan'; locality: Dosang environs, fixed sands; decimalLatitude: 46.92; decimalLongitude: 47.92; samplingProtocol: sand sifting; 
eventDate: 2006-10-7/11; individualCount: 1; recordedBy: A. V. Frolov, L. A. Akhmetova; collectionID: urn:Isid:biocol.org:col:34969; institutionCode: ZIN; collectionCode: Coleoptera

Feeds on: Cattle and wild herbivore dung.

Distribution: This species is thought to be widely distributed in the Holarctic Region. However, Wilson and Angus (2006) demonstrated that the nominative subspecies, $A$. vittatus vittatus Say, 1825, occurring in North America, has a karyotype different from that of Palearctic subspecies $A$. vittatus mundus Reitter, 1892, A. vittatus sellatus Mannerheim, 1852, and $A$. vittatus tjanshanicus Balthasar, 1956. Therefore, A. vittatus can be a complex of closely related species. In the Lower Volga region, A. vittatus mundus occurs.

\section{Cnemisus rufescens (Motschulsky, 1845)}

\section{Materials}

a. country: Russia; stateProvince: Astrakhan'; locality: Dosang environs, right bank of Akhtuba River, floodplain; decimalLatitude: 46.90; decimalLongitude: 47.90; samplingProtocol: hand collecting from marmot hole; eventDate: 2007-04-22; individualCount: 30; recordedBy: A. V. Frolov, L. A. Akhmetova; collectionID: urn:Isid:biocol.org:col:34969; institutionCode: ZIN; collectionCode: Coleoptera

b. country: Russia; stateProvince: Astrakhan'; locality: Dosang environs, right bank of Akhtuba River, floodplain; decimalLatitude: 46.90; decimalLongitude: 47.90; samplingProtocol: hand collecting from marmot hole; eventDate: 2008-04-3/9; individualCount: 47; recordedBy: A. V. Frolov, L. A. Akhmetova; collectionID: urn:Isid:biocol.org:col:34969; institutionCode: ZIN; collectionCode: Coleoptera

Distribution: Caspian lowland desert.

\section{Discussion}

To date, 44 species of the Aphodiini have been recorded from Dosang and its environs. This is apparently the richest recorded local Aphodiini fauna in Russia (by "local fauna" here we understand the fauna occurring in a small area and consisting of sympatric and parapatric populations of different species). The large number of the Aphodiini species found in the area can be explained by a combination of the following factors:

1. Long vegetative season with high effective heat sum. The beetles were found active from March to November and it is possible that some species may be active during winter months, especially during mild winters. The climate in the area is arid in general but the high level of groundwater in sands, even in the driest months, provides suitable conditions for psammophilous taxa.

2. Large livestock including horses and cows provide abundant food resources throughout the year. In the hottest months however, much of the fresh dung is consumed by the Scarabaeinae dung-beetles, notably Scarabaeus typhon FischerWaldheim, and Gymnopleurus mopsus (Pallas). 
3. The collecting area is situated in the transition belt between two floristic districts: Volga-Akhtuba Floodplain (intrazonal) and Desert (zonal) of Caspian Subprovince of Turanian Province (Laktionov 2010).

Apparently due to the last factor, the fauna consists of the two components of different origin. Intrazonal habitats are suitable for a large number of mesophilous species which constitute the core Dosang Aphodiini fauna. These species are widely distributed in the Palearctic region: $A$. bimaculatus, $A$. coenosus, $A$. frater, $A$. ictericus, $A$. kraatzi, $A$. lividus, A. lugens, A. punctipennis, A. quadriguttatus, A. satellitius, A. serotinus, A. testudinarius, $A$. varians, $A$. distinctus, $A$. erraticus, $A$. fimetarius, $A$. granarius, $A$. immundus, $A$. melanostictus, A. rufipes, A. rufus, A. scrofa, A. sturmi, A. subterraneus, $A$. sus, and $A$. vittatus.

Another part of the fauna comprises species with the ranges mostly limited to the steppe, semidesert, and desert zones. These are the species with sethian ( $A$. badenkoi, $A$. clathratus, A. digitalis, A. hauseri, A. kizeritskyi, A. kisilkumi, A. multiplex), westscythiannorthturanian ( $A$. caspius, A. gregarius, A. gresseri, A. zangi, A. curtulus), northturanian plane ranges ( $A$. dosangi, $A$. plustschewskii, $A$. variicolor, $C$. rufescens), and 2 species $(A$. aequalis and $A$. hydrochaeris) distributed in Mediterranean and Central Asia. In Russia, most of these species occur only in the Lower Volga region.

\section{Acknowledgements}

We thank V. P. Bulychev (Dosang Antiplague Station, Dosang) for logistics assistance over many years. We also thank A. V. Ivanov (Institute of Plant and Animal Ecology, Ekaterinburg) and K. V. Makarov and A. V. Matalin (Moscow State Pedagogical University, Moscow) for providing additional specimens. Alberto Ballerio (Brescia), Bruce Gill (Ottawa), and Jan Krikken (Leiden) helped to correct and improve the draft manuscript. This study was supported by the Russian Foundation for Basic Research (grants 07-04-00482, 10-04-00539, and 13-04-010020).

\section{References}

- Akhmetova L (2011) Beetles of the tribe Aphodiini (Coleoptera, Scarabaeidae) of Russia. Dissertation to fulfill the requirement for the degree of Doctor of Philosophy. Zoological Institute RAS, Sankt-Petesburg, 299 pp. [In Russian].

- Akhmetova L, Frolov A (2008a) New records of Aphodius species from Russia (Coleoptera: Scarabaeidae). Zoosystematica Rossica 17: 72. [In English].

- Akhmetova L, Frolov A (2008b) A Review of Scarabs of the Subgenus Nobius Mulsant et Rey, Genus Aphodius Illiger (Coleoptera, Scarabaeidae), of the Fauna of Russia and Neighboring Countries. Entomological Review 88 (4): 421-434. [In English]. DOI: 10.1134/ S0013873808040052

- Akhmetova L, Frolov A (2009) New to Russia and little known species of the genus Aphodius Illiger (Coleoptera, Scarabaeidae). Zoosystematica Rossica 18 (2): 278-284. [In English]. 
- Emeljanov A (1974) Proposals on the classification and nomenclature of areals. Entomologicheskoe Obozrenie 53 (3): 497-522. [In Russian].

- Frolov A (2002) A Review of Aphodiines of the Subgenus Chilothorax Motschulsky, Genus Aphodius Illiger (Coleoptera, Scarabaeidae), from Russia and Neighboring Countries. Entomological Review 82 (1): 1-18. [In English].

- Frolov A (2009) Larval morphology of Aphodius sus (Herbst) and A. variicolor Koshantschikov (Coleoptera: Scarabaeidae: Aphodiinae). Zootaxa 2169: 45-54.

- Kabakov O, Frolov A (1996) A Review of the Beetles of the Genus Aphodius III. (Coleoptera, Scarabaeidae) from Russia and Adjacent Countries, Related to the Subgenus Acrossus Muls. Entomological Review 76 (9): 1165-1181. [In English].

- Laktionov A (2010) Flora Astrakhanskoj Oblasti. [Flora of Astrakhan Province]. Astrakhanskij Universitet, Astrakhan, 296 pp. [In Russian].

- Nikolajev G (1987) Plastinchatousye zhuki Kazakhstana i Srednej Azii. [Scarab beetles of Kazakhstan and Middle Asia]. Nauka, Alma-Ata, 232 pp. [In Russian].

- Shokhin I (2007) Contribution to the fauna of lamellicorn beetles (Coleoptera, Scarabaeoidea) of Southern Russia, with some nomenclatural changes in the family Scarabaeidae. Caucasian Entomological Bulletin 3 (2): 105-185. [In Russian].

- Wilson C, Angus R (2006) A chromosomal analysis of eight species of Aphodius Illiger, subgenera Agiolinus Schmidt, Agrilinus Mulsant \& Rey and Planolinus Mulsant \& Rey (Coleoptera: Aphodiidae). Proceedings of the Russian Entomological Society 77: 28-33.

\section{Supplementary material}

\section{Suppl. material 1: Occurences data for checklist of Aphodiini of Dosang environs}

Authors: Frolov, A. V., Akhmetova, L. A.

Data type: occurences

Brief description: Supplementary file with occurences data for cheklist of Aphodiini of Dosang environs (Astrakhan Province, European Russia) mostly collected by A. V. Frolov and L. A. Akhmetova in 2006-2012.

Filename: Species_occurrence-1_v1_DwC_Dosang.xls - Download file $(208.00 \mathrm{~kb})$ 